\title{
THE UNDECIDABILITY OF THE RECURSIVELY ENUMERABLE DEGREES
}

BY LEO HARRINGTON AND SAHARON SHELAH ${ }^{1}$

Let $\leqslant$ be Turing reducibility between subsets of $\omega$ and let $R$ be the collection of all recursively enumerable subsets of $\omega$. For $A$ in $\mathrm{R}, \mathrm{A}$ is the Turing degree of $A$; and $\mathrm{R}=\{\mathbf{A} ; A$ is in $\mathrm{R}\}$. (See [2] for any unexplained notions or notation used above, or below). We also identify $R$ with the structure $\langle R, \leqslant\rangle$.

We wish to announce the result

THEOREM. The first order theory of $R$ is undecidable.

To prove this we show that the theory of partial orderings is reducible to the theory of $R$, as follows.

Recall, $R$ is an upper-semi-lattice; thus for $a, b$ in $R$, their join $a \vee b$ is in $R$.

Lemma. For Pa partial ordering recursive in (say) $0^{\prime}$, there are $a, b_{p}$ $(p \in P), c, d$, $e$ in $R$ such that

(i) $b_{p} \leqslant a$,

(ii) $a \leqslant\left(b_{p} \vee C\right)$,

(iii) for each $Z \leqslant a$, either

( $\alpha) a \leqslant(Z \vee c)$, or $\exists p \in P$ such that

$(\beta)_{p} Z \leqslant\left(b_{p} \vee d\right)$,

(iv) for $p \neq q, b_{p} \nless\left(b_{q} \vee d\right)$,

( ) for $p, q$ in $P, p \leqslant q$ iff $b_{p} \leqslant\left(b_{q} \vee d \vee e\right)$.

Now, for $a, b, c, d$ in $R$ let $\phi(a, b, c, d) \equiv(b \leqslant a)$ and $(a \nless(b \vee c))$ and $(\neg E Z(Z \leqslant a$ and $a \nless(Z \vee c)$ and $(Z \vee d)>(b \vee d)))$. For $a, c, d$, $e$ in $R$, let $Q(a, c, d, e)=\{b \vee d \vee e ; R \vDash \phi(a, b, c, d)\}$. For $a, c, d, e$ as in the lemma, $Q(a, c, d, e)=\left\{b_{p} \vee d \vee e ; p \in P\right\}$, and $\langle Q(a, c, d, e), \leqslant\rangle$ is isomorphic to $P$.

Thus for $\psi$ a sentence of the language of partial orderings: $\psi$ is true of some partial ordering iff (by the usual proof of the completeness theorem) $P \vDash \psi$ for some $P$ recursive in $0^{\prime}$ iff (by the lemma) $\exists a, c, d, e$ in $R$ $(\langle Q(a, c, d, e), \leqslant\rangle \vDash \psi)$.

The lemma is proven, of course, by a priority argument. The type of priority argument used can best be described as an infinite injury argument with a finite injury priority argument on top of it. This kind of construction was first

Received by the editors February 3, 1981.

AMS (MOS) subject classifications (1970). Primary 02F25, 02 F30.

1 The authors were supported by the NSF. 
used by Lachlan [1], and is now (for reasons best not discussed here) called a monstrous injury argument. We briefly describe the construction which proves the lemma.

As in most priority arguments, we will have requirements which attempt to satisfy various instances of (i)-(v). Each requirement is provided with a guess concerning certain stronger requirements. Each guess involves a finite amount of $\Delta_{3}^{0}$ information, and thus an initial segment of $0^{\prime \prime}$. By an exhaustive guessing procedure, all correct guesses are made (in addition to may incorrect guesses), and by an infinite injury type procedure, the requirements with incorrect guesses do not really interfer with those with correct guesses. Thus, by ignoring the requirements with incorrect guesses, we are left with a construction in which each requirement behaves recursively, but in which both the list of requirements, and how the requirements behave, are picked recursively in $0^{\prime \prime}$. So, in some sense, the construction is now done recursively in $0^{\prime \prime}$; the $n$th stage of the construction now involves picking the $n$th requirement, and finding the answers to certain $\Delta_{3}^{0}$ questions concerning the first $n$ requirements. (The most typical questions asked of a requirement are: "Is it working?" or "Has it failed?".) From this point of view, the construction is now a finite injury argument. The finite injury comes about when trying to satisfy an instance of (iii) (say the instance for $z=Z$ ): we will first attempt to make (iii) ( $\alpha$ ) true via a requirement $R$; if $R$ later interfers with some other requirement $S$ (it can only interfer with an $S$ trying to satisfy an instance of (ii)), then we will kill off $R$ (i.e., $R$ attempts to build a reducibility demonstrating that $a \leqslant(Z \vee c) ; R$ is killed off by making this reducibility partial) and create a requirement $\hat{R}$ which attempts to ensure (iii) $(\beta)_{p}$ (where $S$ works on (ii) $)_{p}$ ). The killing off of $R$ can interfer with requirements stronger than $S$ but weaker than $R$; in other words, these requirements are injured, and this leads to the finite injury nature of the construction. The finiteness of these injuries is ensured by arranging that $\hat{R}$ cannot interfer with any requirement. (Intuitively, weaker requirements than $\hat{R}$ will use the fact that $S$ failed because of $R$. Upon examination, this means that at certain crucial stages (here stages refer to the recursive construction, not the construction recursive in $0^{\prime \prime}$ ), numbers failed to enter $Z$; but $\hat{R}$ will cause difficulties only when numbers enter $Z$. Thus if, by arrangement, the only stages at which $\hat{R}$ can interfer with another requirement are those above-mentioned crucial stages, then $\hat{R}$ will be prevented from interfering.)

\section{REFERENCES}

1. A. H. Lachlan, A recursively enumerable degree which will not split over all lesser ones, Ann. Math. Logic 9 (1975), 307-365.

2. H. Rogers, Jr., Theory of recursive functions and effective computability, McGrawHill, New York, 1967.

HEBREW UNIVERSITY, INSTITUTE FOR ADVANCED STUDIES, MOUNT SCOPUS, JERUSALEM, ISRAEL 